\title{
Association between metabolic syndrome and bone mineral density among menopausal Saudi women: Case-control study
}

\author{
Essra Al-Dawood ${ }^{1}$, Mubashir Zafar*2 (D) \\ Received: 14 May 2020 \\ Published: 18 Feb 2021
}

\begin{abstract}
Background: Metabolic syndrome (MetS) and osteoporosis are two of the world's major healthcare issues. There are several studies which explored the association between MetS and bone mineral density (BMD), but all of them are cross-sectional. These studies cover all populations, including expatriated, which did not determine the actual problem among Saudi women. This is the first casecontrol study that determines the causal relationship between MetS and BMD. The objective of this study is to determine the relationship between metabolic syndrome and bone mass density among Saudi menopausal women in Eastern Province - Saudi Arabia.

Methods: It's a case-control study and 380 menopausal Saudi women were selected through simple random sampling. They were divided into 190 cases with osteoporosis and 190 without osteoporosis. Bone Mineral Density (BMD) at the total hip was determined using dual-energy X-ray absorptiometry (DEXA). T score was calculated. The association between the risk factors of MetS and bone mineral density was determined by binary logistic regression analysis using SPSS (statistical package of social science) software.

Results: Among women, the prevalence of MetS was substantially higher in those with osteoporosis. The Mets is positively correlated with bone mineral density. ( $\mathrm{r}=0.08, \mathrm{p}=0.051)$. The occurrence of MetS was associated with increased osteoporosis among Saudi women $(B=0.004 ; p=0.005)$ after adjustment of confounders. The existence of obesity was significantly associated with increased odds of Bone marrow density among women (OR 2.56; $95 \% \mathrm{CI}, 2.22-3.44 ; \mathrm{p}=0.030$ ) after adjustment of confounders.
\end{abstract}

Conclusion: The incidence of MetS was associated with osteoporosis in Saudi women.

Keywords: Bone mineral density, Menopausal women, Metabolic syndrome, Osteoporosis

Conflicts of Interest: None declared

Funding: None

*This work has been published under CC BY-NC-SA 1.0 license.

Copyright $₫$ Iran University of Medical Sciences

Cite this article as: Al-Dawood E, Zafar M. Association between metabolic syndrome and bone mineral density among menopausal Saudi women: Case-control study. Med J Islam Repub Iran. 2021 (18 Feb);35:26. https://doi.org/10.47176/mjiri.35.26

\section{Introduction}

The occurrence of metabolic syndrome (MetS) is expanding at a very high rate in developed and developing nations throughout the world (1). MetS is a cluster of risk factors of heart disease such as high blood pressure, high blood cholesterol level, high waist circumference, raised fasting plasma glucose and abdominal obesity (2). Epide-

Corresponding author: Dr Mubashir Zafar, researchmubshir@outlook.com

1. Primary Health Care Centre, Ministry of Health, Al-Jubail, KSA

2. Department of Family and Community Medicine, College of Medicine, University of Hail, Hail, KSA miologic researches indicate that metabolic syndrome is the most important risk factor for various numbers of chronic diseases mainly heart-related diseases and type II diabetes (3). It is estimated that the prevalence of MetS ranges from $20 \%$ to $25 \%$ of the population in the world. (3) A study found that people with MetS likely to die

\section{$\uparrow$ What is "already known" in this topic:}

There are several studies that determine the relationship between MetS and BMD, but all of them are cross-sectional; These studies cover all populations including expatriated which did not determine the actual problem among Saudi women.

\section{$\rightarrow$ What this article adds:}

This is the first case-control study that determines the causal relationship between MetS and BMD. This study evaluates only Saudi females who were included as survey respondents. The study results found a positive relationship between MetS and BMD and obesity is an important predictor for BMD. 
more than twice compared to individuals with no syndrome. (3) A survey published in 2018 shows that in Saudi Arabia, the prevalence of MetS is approximately $39.8 \%$. (4) It is estimated that 8.9 million fractures yearly are reported among 200 million people; it means every 3 seconds, one fracture is reported. (5) A study conducted previously and the findings showed that waist circumference is significantly related to body surface area (BSA) $(\mathrm{r}=0.186, \mathrm{p}<0.01)$. This result was similar to a crosssectional study (6) that included 2007 participants (1045 males and 962 females) over 50 years of age to examine the link between MetS and osteoporosis. Another study was conducted in China showed similar results and a positive association of MetS with osteoporosis among elderly populations. (7).

Although MetS and osteoporosis were earlier thought to be two unrelated diseases, studies have shown that both conditions share several genetic, nutritional, and hormonal factors (8). There are different studies that determine the association of MetS with osteoporosis, but the specific association between MetS risk factors never determined. (9-15).

The prevalence of metabolic syndrome has increased in Saudi Arabia, forcing such a need to determine the causal factors leading to MetS among the aged population. Life expectancy also rises, which raises Saudi women's risk of osteoporosis. Only limited studies have focused on explaining the relationship between MetS and BMD in Saudi Arabia. These studies cover all populations including expatriated which did not determine the actual problem among Saudi women. These studies are cross-sectional and did not determine the temporality (causal association). This research will also be the first case-control study undertaken in Saudi Arabia in order to determine a possible causal relationship between the two conditions. Furthermore, only Saudi females will be included as survey respondents estimating the real issue among Saudi women.

The objective of this study is to determine the association between MetS and BMD among Saudi menopausal women in Eastern Province - Saudi Arabia.

\section{Methods}

\section{Study setting}

This case-control study was performed at Safwa General Hospital, one of the Eastern Province's main government facilities consisting of nearly 30 beds and serving approximately $150-200$ patients daily in the outpatient departments (OPD). And also, patients will be included from one of the biggest primary healthcare centers in Qatif that serve about 80-100 patients daily.

\section{Study participants}

All menopausal women (age 45-75 years old) who had a BMD scan of Dual-Energy X-ray Absorptiometry (DEXA) (Lunar Prodigy Advance; GE Healthcare, Madison, WI, USA) and were diagnosed as osteoporotic were included as cases and women without osteoporosis were included as controls. The patients were referred by their physicians after visiting the hospital for regular checkups.

Menopausal status was determined based on the history of lack of menstruation for the last one year.

Inclusion criteria for cases: All menopausal women between the age of 45-75 years and clinically confirmed by their physician using a BMD scan were included. All were diagnosed with MetS.

Exclusion criteria: Peri-menopausal women, participants with other co-morbidity and women under hormonal treatment were excluded.

Inclusion criteria for controls: All menopausal women aged 45 and 75 years old who were free of osteoporosis with MetS were included.

Exclusion criteria: Perimenopausal women, age above 75 years or patients receiving estrogen replacement therapy were excluded. Hormonal replacement therapy was defined as the therapeutic use of hormones typically to increase diminished levels in the body.

\section{Sample size}

Sample size was calculated by WHO sample size calculator for health studies. Based on 95\% confidence level, a relative precision of 0.25 , the probability of the exposure to disease of 0.6 , the probability of the exposure to no disease of 0.4 , and anticipated odds ratio of 1.5 , the sample size of this study was 380 . The sample size was equally divided into 190 participants for the cases and 190 for the controls. The participants were selected through simple random sampling.

\section{Data collection tool and technique}

Structured questionnaire was used. The questionnaire was divided into four sections, including sociodemographic characteristics, anthropometric measurement, biochemical measurements including FBG, RBG, lipid profile, and DEXA measurement.

\section{Anthropometry, blood sample and analysis}

Anthropometric measures were determined through an automatic Anthrop Meter. Body mass index (BMI) was calculated through the formula, weight $(\mathrm{Kg})$ divided by height $\left(\mathrm{m}^{2}\right)$; and then classified according to the WHO classes: Normal weight $\left(18.5-24.99 \mathrm{Kg} / \mathrm{m}^{2}\right)$, Overweight $\left(25-29.99 \mathrm{Kg} / \mathrm{m}^{2}\right)$, Obese $\left(\geq 30 \mathrm{Kg} / \mathrm{m}^{2}\right)$, the waist circumference (WC) was calculated using a tape.

Blood pressure was calculated using a blood pressure apparatus.

Blood samples were collected after 8 to 12 hours of fasting to meausre Fasting Blood Sugar (FBS), High Density Lipoprotein (HDL), and Triglyceride (TG).

\section{Criteria for the diagnosis of metabolic syndrome}

Participants were diagnosed with MetS using the criteria (at least three components):

1. Waist circumference: (WC) $\geq 102 \mathrm{~cm}$ (males), $\geq 88$ cm (females), 2. Hyperglycemia: fasting blood sugar (FBS) $\geq 100 \mathrm{mg} / \mathrm{dl}, 3$. Hypertriglyceridemia: serum triglycerides $(\mathrm{TG}) \geq 150 \mathrm{mg} / \mathrm{dl}$, 4. Serum HDL-cholesterol (HDL-C) $<50 \mathrm{mg} / \mathrm{dl}$, 5. Systolic blood pressure (SBP) $\geq$ $130 \mathrm{mmHg}$ and/or diastolic blood pressure (DBP) $\geq 85$ $\mathrm{mmHg}$. 


\section{Criteria for diagnosis of osteoporosis}

The bone mineral density of the total hip was measured using dual-energy X-ray absorptiometry (DEXA). The T scores were calculated, and the diagnosis of osteoporosis was made according to the World Health Organization criteria: Osteopenia ( $\mathrm{T}$ score between from -1 to -2.5 ), Osteoporosis (T score $<-2.5$ ) and Normal (T score $>-1$ ).

T-Score Tertiles: T score was divided into tertiles (tertile 1: low score, tertile 3: high score).

\section{Ethical consideration}

Ethical approval was received from Imam Abdulrahman Bin Faisal University research ethical review board. Permission was taken from the hospital. Informed consent was obtained from all subjects who agreed to participate in the study before the interview. Participation was voluntary, and participants were free to withdraw at any time without any explanation. The confidentiality and privacy of the subjects were maintained and there was no financial benefit to either the subjects or the researcher.

\section{Statistical analysis}

All Statistical analysis was performed with SPSS win- dows version 23. Continuous data were presented as mean \pm standard deviation (SD). Statistical significance between the groups was evaluated using Chi-square test. Correlation between MetS and BMD was determined using Pearson's correlation test. A Confident Interval (CI) of 95\% and a P-value of less than 0.05 (two-sided) was considered as statistically significant. Binary logistic regression was used to determine the association between MetS and BMD. T-score tertile was used as a dependent variable (with the lowest tertile as a reference) and full MetS or its individual components (present vs. absent) as independent variables.

\section{Results}

The mean age of is the participants were $63.8 \pm 7.1$ years. The data showed a statistically significant difference in age categories between women with MetS and osteoporosis compared to their counterparts, i.e. those with osteoporosis who were older $(\mathrm{p}=0.000)$ (Table 1$)$.

Prevalence of osteoporosis was higher among those women who were physically inactive $(57.9 \%$ vs. 42.1 ; $\mathrm{p}=0.016$ compared to those without osteoporosis $(58.3 \%$ vs. $41.7 \%) ; \mathrm{p}=0.016)$.

\begin{tabular}{|c|c|c|c|c|}
\hline $\begin{array}{l}\text { Sr. } \\
\text { No }\end{array}$ & Characteristics & $\begin{array}{c}\text { Cases }=190 \\
\mathrm{~N}(\%)\end{array}$ & $\begin{array}{c}\text { Control=190 } \\
\text { N }(\%)\end{array}$ & $\mathrm{p}^{*}$ \\
\hline 1 & & $65.45 \pm 7.24$ & $62.15 \pm 7.12$ & \\
\hline 2 & $\begin{array}{l}\text { Age }(\text { years })(\text { Mean } \pm S D) \\
\text { Age Categories } \\
45-60 \text { years }\end{array}$ & $59(36.1)$ & $59(63.9)$ & \\
\hline & $61-75$ years & $131(56.3)$ & $131(43.7)$ & 0.001 \\
\hline 3 & Age at Menopause (years) (Mean \pm SD) & $51.70 \pm 4.97$ & $51.35 \pm 3.94$ & \\
\hline 4 & $\begin{array}{l}\text { Marital status } \\
\text { Single } \\
\text { Married } \\
\text { Widowed\& Divorced }\end{array}$ & $\begin{array}{c}8(46.7) \\
141(48.6) \\
41(55.4)\end{array}$ & $\begin{array}{c}8(53.3) \\
141(51.4) \\
41(44.6)\end{array}$ & 0.530 \\
\hline 5 & $\begin{array}{l}\text { Education level } \\
\text { Illiterate } \\
\text { Intermediate } \\
\text { High School\& University }\end{array}$ & $\begin{array}{l}76(53.3) \\
67(50) \\
47(44.7)\end{array}$ & $\begin{array}{l}76(46.7) \\
67(50) \\
47(55.3)\end{array}$ & 0.423 \\
\hline 6 & $\begin{array}{l}\text { Occupation } \\
\text { Housewife } \\
\text { Employed }\end{array}$ & $\begin{array}{c}173(51) \\
17(40)\end{array}$ & $\begin{array}{c}173(49) \\
17(60)\end{array}$ & 0.214 \\
\hline 7 & $\begin{array}{l}\text { Exposure to Sunlight per day } \\
\text { No } \\
\text { Yes }\end{array}$ & $\begin{array}{c}110(51.6) \\
80(47.8)\end{array}$ & $\begin{array}{l}110(48.4) \\
80(52.2)\end{array}$ & 0.467 \\
\hline 8 & $\begin{array}{l}\text { Covering hair only } \\
\text { Eyes shown only } \\
\text { Full cover }\end{array}$ & $\begin{array}{l}\text { Veil Type } \\
20(53.8) \\
108(49.3) \\
62(50)\end{array}$ & $\begin{array}{c}20(46.2) \\
108(50.7) \\
62(50)\end{array}$ & 0.873 \\
\hline 9 & $\begin{array}{l}\text { Physical Activity } \\
\text { No } \\
\text { Yes , 1-2 times/day } \\
>3 \text { times/ day }\end{array}$ & $\begin{array}{l}85(57.9) \\
66(41.7) \\
39(46.8)\end{array}$ & $\begin{array}{l}85(42.1) \\
66(58.3) \\
39(53.2)\end{array}$ & 0.016 \\
\hline 10 & $\begin{array}{l}\text { Smoking } \\
\text { Never smoke } \\
\text { Ever smoke }\end{array}$ & $\begin{array}{c}103(49.5) \\
87(50.6)\end{array}$ & $\begin{array}{c}103(50.5 \%) \\
87(49.4 \%)\end{array}$ & 0.837 \\
\hline 11 & $\begin{array}{l}\text { Family History of Met } \\
\text { Yes } \\
\text { No }\end{array}$ & $\begin{array}{c}145(51.4) \\
45(45.6)\end{array}$ & $\begin{array}{c}145(48.6) \\
45(54.4)\end{array}$ & 0.334 \\
\hline
\end{tabular}

Data are given as the mean \pm SD or as the number of subjects with percentages given in parentheses, as appropriate. Categorical data are compared by $\chi 2$ test, BMD: bone mineral density. P-value $<0.05$ significant. 
Table 2 shows the difference in anthropometric and biochemical parameters of all participants. Osteoporosis was significantly less common among women with higher BMI compared to their counterparts (58\% vs.42\%, $\mathrm{p}=0.001)$. In addition, participants with higher Serum TG level had significantly higher number of osteoporosis compared with their counterparts $(p=.000)$. Moreover, women with lower mean levels of HDL-C had significantly less cases of osteoporosis compared to their counterparts $(\mathrm{p}<0.001)$

A low BMD represented a T1-score and a high BMD represented T3-score. Different components of MetS showed no difference among various tertiles in both groups. The data does not show any significant statistical difference in either group (Table 3).

A very weak negative correlation was observed between BMD and SBP $(\mathrm{r}=-0.072, \mathrm{p}=0.320)$, TG $(\mathrm{r}=-0.069$, $\mathrm{p}=0.342)$ and HDL $(r=-.065, \mathrm{p}=0.375)$ in women with osteoporosis, and between BMD and WC $(\mathrm{r}=-0.091$, $\mathrm{p}=0.213)$, TG $(\mathrm{r}=-0.025, \mathrm{p}=0.729)$ and HDL $(\mathrm{r}=-0.061$, $\mathrm{p}=0.406)$ in their control counterparts. No statistical significance was found in either group ( $\mathrm{p}>0.05$ ) (Table 4).

According to Table 5, all the socio-demographic characteristics were associated with osteoporosis but none of them were statistically significant. As Table 6 depicts, triglyceride is the only component of metabolic syndrome

\begin{tabular}{|c|c|c|c|c|}
\hline S. no & Characteristics & $\begin{array}{c}\text { Cases }=190 \\
\mathrm{~N}(\%) \\
\end{array}$ & $\begin{array}{c}\text { Control }=190 \\
\mathrm{n}(\%)\end{array}$ & $\mathrm{p}$ \\
\hline 1 & $\begin{array}{l}\text { Mean Height }(\mathrm{cm}) \\
(\text { Mean } \pm \text { SD) }\end{array}$ & $153.20 \pm 9.57$ & $155.46 \pm 7.08$ & - \\
\hline 2 & $\begin{array}{l}\text { Mean Weight }(\mathrm{kg}) \\
(\text { Mean } \pm \text { SD) }\end{array}$ & $69.71 \pm 16.76$ & $78.05 \pm 16.86$ & - \\
\hline 3 & Mean WC (cm) & & & \\
\hline & $\begin{array}{l}\text { Normal }(\mathrm{WC}<88 \mathrm{~cm}) \\
\text { Obese }(\mathrm{WC} \geq 88 \mathrm{CM})\end{array}$ & $\begin{array}{l}43(58.1 \%) \\
147(47.6 \%)\end{array}$ & $\begin{array}{l}43(41.9 \%) \\
147(52.4 \%)\end{array}$ & 0.086 \\
\hline 4 & $\begin{array}{l}\text { BMI }\left(\mathrm{kg} / \mathrm{m}^{2}\right) \\
(\text { Mean } \pm \mathrm{SD})\end{array}$ & $31.26 \pm 22.33$ & $32.15 \pm 6.37$ & - \\
\hline 5 & $\begin{array}{l}\text { BMI class } \\
\text { Normal } \\
\text { Under weight } \\
\text { Overweight } \\
\text { Obese }\end{array}$ & $\begin{array}{l}30(67.2 \%) \\
2(100 \%) \\
61(52.8 \%) \\
97(42 \%)\end{array}$ & $\begin{array}{l}31(32.8 \%) \\
0(0 \%) \\
62(47.2 \%) \\
97(58 \%)\end{array}$ & 0.001 \\
\hline 6 & $\begin{array}{l}\text { Mean SBP }(\mathrm{mmHg}) \\
\text { Normal }(<130 \mathrm{mmHg}) \\
\text { High }(\geq 130 \mathrm{mmHg})\end{array}$ & $\begin{array}{c}154(50.2 \%) \\
36(49.3 \%)\end{array}$ & $\begin{array}{l}154(49.8 \%) \\
36(50.7 \%)\end{array}$ & 0.896 \\
\hline 7 & $\begin{array}{l}\text { Mean DBP }(\mathrm{mmHg}) \\
\text { Normal }(<85 \mathrm{mmHg}) \\
\operatorname{High}(\geq 85 \mathrm{mmHg})\end{array}$ & $\begin{array}{c}44(51.7 \%) \\
146(49.5 \%)\end{array}$ & $\begin{array}{c}44(48.3 \%) \\
146(50.5 \%)\end{array}$ & 0.714 \\
\hline 8 & $\begin{array}{l}\text { Level of Triglyceride (TG) } \\
\text { Normal }(<90 \mathrm{mg} / \mathrm{dl}) \\
\text { Borderline }(90-129 \mathrm{mg} / \mathrm{dl}) \\
\text { High }(>129 \mathrm{mg} / \mathrm{dl})\end{array}$ & $\begin{array}{c}78(41 \%) \\
54(40.4 \%) \\
58(71.3 \%)\end{array}$ & $\begin{array}{c}78(59 \%) \\
54(59.6 \%) \\
58(28.7 \%)\end{array}$ & 0.000 \\
\hline 9 & $\begin{array}{l}\text { Level of High-Density Lipoprotein (HDL) } \\
\text { Normal }(>45 \mathrm{mg} / \mathrm{dl}) \\
\text { Borderline }(40-45 \mathrm{mg} / \mathrm{dl}) \\
\text { Low }(<40 \mathrm{mmol} / \mathrm{L})\end{array}$ & $\begin{array}{l}129(43 \%) \\
29(64.9 \%) \\
32(64.6 \%)\end{array}$ & $\begin{array}{l}129(57 \%) \\
29(35.1 \%) \\
32(35.4 \%)\end{array}$ & 0.000 \\
\hline 10 & $\begin{array}{l}\text { Fasting blood Sugar } \\
\text { Normal }(<100 \mathrm{mg} / \mathrm{dl}) \\
\text { Borderline }(100-125 \mathrm{mg} / \mathrm{dl}) \\
\text { High }>125 \mathrm{mg} / \mathrm{dl})\end{array}$ & $\begin{array}{l}41(50.6 \%) \\
79(51.9 \%) \\
70(47.5 \%)\end{array}$ & $\begin{array}{l}41(49.5 \%) \\
79(48.1 \%) \\
70(52.5 \%)\end{array}$ & 0.744 \\
\hline 11 & $\begin{array}{l}\text { T-Score } \\
\text { T1 (Lowest) } \\
\text { T2 } \\
\text { T3 (Highest) }\end{array}$ & $\begin{array}{c}50(100 \%) \\
93(47.8 \%) \\
47(0 \%)\end{array}$ & $\begin{array}{c}50(0 \%) \\
93(52.2 \%) \\
47(100 \%) \\
\end{array}$ & 0.000 \\
\hline
\end{tabular}

Categorical data are compared by $\chi 2$ test. WHO criteria: a T-score between -1 and -2.5 is indicative of osteopenia, while a T-score of -2.5 and below reflects osteoporosis; a $\mathrm{T}$-score of -1 and above is considered normal. $\mathrm{P}$-value $<0.05$ significant.

Table 3. Prevalence of metabolic syndrome (MetS) components in tertile of T-score $(\mathrm{n}=380)$

\begin{tabular}{|c|c|c|c|c|c|c|c|}
\hline \multirow[t]{2}{*}{ S.no } & \multirow[b]{2}{*}{ Components of Metabolic Syndrome } & \multicolumn{3}{|c|}{ Cases $(n=190)$} & \multicolumn{3}{|c|}{ Control $(n=190)$} \\
\hline & & $\mathrm{T} 1$ & $\mathrm{~T} 2$ & $\mathrm{p}$ & $\mathrm{T} 2$ & T3 & $\mathrm{p}$ \\
\hline 1 & Obesity & $50.7 \%$ & $42.5 \%$ & 0.340 & $53.2 \%$ & $64.8 \%$ & 0.211 \\
\hline 2 & Hyperglycemia & $52.5 \%$ & $47.5 \%$ & 0.675 & $49.7 \%$ & $50.3 \%$ & 0.357 \\
\hline 3 & Low HDL level & $55.3 \%$ & $44.7 \%$ & 0.372 & $54.7 \%$ & $45.3 \%$ & 0.403 \\
\hline \multirow[t]{3}{*}{4} & Hypertension & & & & & & \\
\hline & SPB & $55.2 \%$ & $44.8 \%$ & 0.318 & $47.3 \%$ & $52.7 \%$ & 0.052 \\
\hline & $\mathrm{DPB}$ & $44.4 \%$ & $55.6 \%$ & 0.245 & $48.6 \%$ & $51.4 \%$ & 0.744 \\
\hline 5 & Hypertriglyceridemia & $57.5 \%$ & $46.8 \%$ & 0.340 & $54.5 \%$ & $45.5 \%$ & 0.727 \\
\hline
\end{tabular}

Categorical data are compared by $\chi 2$ test. WHO criteria: a T-score between -1 and -2.5 is indicative of osteopenia, while a T-score of -2.5 and below reflects osteoporosis; a T-score of -1 and above is considered normal. P-value $<0.05$ significant. 


\begin{tabular}{|c|c|c|c|}
\hline S.no & Variables & BMD cases & BMD controls \\
\hline & & T score & T score \\
\hline & & $\begin{array}{c}\mathrm{r} \\
(\mathrm{p})\end{array}$ & $\begin{array}{c}\mathrm{r} \\
(\mathrm{p})\end{array}$ \\
\hline \multirow[t]{2}{*}{1} & $\mathrm{WC}(\mathrm{cm})$ & .082 & -.091 \\
\hline & & $(0.050)$ & $(0.213)$ \\
\hline \multirow[t]{2}{*}{2} & SBP (mmHg) & -.072 & .141 \\
\hline & & $(0.320)$ & $(0.052)$ \\
\hline \multirow[t]{2}{*}{3} & $\mathrm{DBP}(\mathrm{mmHg})$ & .084 & .024 \\
\hline & & $(0.247)$ & $(0.746)$ \\
\hline 4 & FBS (mmol/L) & $\begin{array}{c}.030 \\
(0.677)\end{array}$ & $\begin{array}{c}.067 \\
(0.360)\end{array}$ \\
\hline 5 & $\mathrm{TG}(\mathrm{mmol} / \mathrm{L})$ & $\begin{array}{c}-.069 \\
(0.342)\end{array}$ & $\begin{array}{c}-.025 \\
(0.729)\end{array}$ \\
\hline 6 & $\mathrm{HDL}(\mathrm{mmol} / \mathrm{L})$ & $\begin{array}{c}-.065 \\
(0.375)\end{array}$ & $\begin{array}{c}-.061 \\
(0.406)\end{array}$ \\
\hline
\end{tabular}

r: Pearson correlations. WC: waist circumference, SBP: systolic blood pressure, DBP: diastolic blood pressure, FBG: fasting blood glucose, TG: triglycerides HDL-C: High-density lipoprotein cholesterol. P-value $<0.05$ significant

\begin{tabular}{|c|c|c|c|c|c|c|}
\hline Risk Factors & $\begin{array}{c}\text { Cases } \\
(\mathrm{n}=190) \\
\mathrm{N}(\%) \\
\end{array}$ & $\begin{array}{c}\text { Control } \\
(\mathrm{n}=190) \\
\mathrm{N}(\%)\end{array}$ & $\begin{array}{c}\text { Unadjusted risk } \\
\text { Estimate } \\
\text { OR }(95 \% \mathrm{CI})\end{array}$ & P-value & $\begin{array}{c}\text { Adjusted Risk } \\
\text { Estimate } \\
\text { OR }(95 \% \mathrm{CI}) \\
\end{array}$ & $\mathrm{p}$ \\
\hline \multicolumn{7}{|l|}{ Age } \\
\hline $45-60$ years & $59.5(36.1 \%)$ & $76(40 \%)$ & $\begin{array}{c}0.439 \\
(0.281-0.686)\end{array}$ & 0.000 & $\begin{array}{c}0.128 \\
(0.050-.327)\end{array}$ & 0.000 \\
\hline $\begin{array}{l}61-75 \text { years } \\
\text { BMI }\end{array}$ & $130.5(56.3 \%)$ & $114(60 \%)$ & $0 \mathrm{~b}$ & $0 \mathrm{~b}$ & $0 \mathrm{~b}$ & $0 \mathrm{~b}$ \\
\hline Normal & $44(23.2 \%)$ & $20(10.5 \%)$ & $0 \mathrm{~b}$ & $0 b$ & ob & $0 \mathrm{~b}$ \\
\hline Overweight & $65(34.2 \%)$ & $58(30.5 \%)$ & $\begin{array}{c}.509 \\
(0.270-.962)\end{array}$ & 0.038 & $\begin{array}{c}1.087 \\
(0.398-2.964)\end{array}$ & 0.871 \\
\hline Obese & $81(42.6 \%)$ & $112(58.9 \%)$ & $\begin{array}{c}1.329 \\
(1.180-1.600)\end{array}$ & 0.000 & $\begin{array}{c}2.569 \\
(2.224-3.445)\end{array}$ & 0.030 \\
\hline \multicolumn{7}{|l|}{ Marital Status } \\
\hline Single & $7(3.7 \%)$ & $8(4.2 \%)$ & $\begin{array}{c}0.704 \\
(0.234-2.120)\end{array}$ & 0.532 & $\begin{array}{c}0.400 \\
(0.059-2.716)\end{array}$ & 0.348 \\
\hline Married & $137(72.1 \%)$ & $145(76.3 \%)$ & $\begin{array}{c}0.760 \\
(.465-1.243)\end{array}$ & 0.274 & $\begin{array}{c}0.797 \\
(0.355-1.789)\end{array}$ & 0.582 \\
\hline $\begin{array}{l}\text { Widowed } \\
\text { Education }\end{array}$ & $46(24.2 \%)$ & $37(19.5 \%)$ & $0 \mathrm{~b}$ & $0 \mathrm{~b}$ & $0 \mathrm{~b}$ & $0 \mathrm{~b}$ \\
\hline Illiterate & $81(42.6 \%)$ & $71(37.4 \%)$ & $\begin{array}{c}1.412 \\
(0.843-2.368)\end{array}$ & 0.190 & $\begin{array}{c}0.702 \\
(0.222-2.227)\end{array}$ & 0.548 \\
\hline Intermediate & $67(35.3 \%)$ & $67(35.3 \%)$ & $\begin{array}{c}1.238 \\
(0.729-2.102)\end{array}$ & 0.429 & $\begin{array}{c}0.865 \\
(0.306-2.447)\end{array}$ & 0.785 \\
\hline $\begin{array}{l}\text { University } \\
\text { Occupation }\end{array}$ & $42(22.1 \%)$ & $52(27.4 \%)$ & $0 \mathrm{~b}$ & $0 \mathrm{~b}$ & $0 \mathrm{~b}$ & $0 \mathrm{~b}$ \\
\hline Employed & $14(7.4 \%)$ & $21(11.1 \%)$ & $0 \mathrm{~b}$ & $0 \mathrm{~b}$ & $0 b$ & $0 \mathrm{~b}$ \\
\hline Unemployed & $176(92.6 \%)$ & $169(88.9 \%)$ & $\begin{array}{c}1.562 \\
(0.769-3.172)\end{array}$ & 0.217 & $\begin{array}{c}1.686 \\
(0.345-8.228)\end{array}$ & 0.519 \\
\hline Veil Type & & & & & & \\
\hline Hair only & $21(11.1 \%)$ & $18(9.5 \%)$ & $0 \mathrm{~b}$ & $0 \mathrm{~b}$ & $0 \mathrm{~b}$ & $0 \mathrm{~b}$ \\
\hline Eyes shown & $107(56.3 \%)$ & $110(57.9 \%)$ & $\begin{array}{c}0.834 \\
(0.421-1.652)\end{array}$ & 0.602 & $\begin{array}{c}0.713 \\
(0.214-2.372)\end{array}$ & 0.581 \\
\hline Full cover & $62(32.6 \%)$ & $62(32.6 \%)$ & $\begin{array}{c}0.857 \\
(0.417-1.763)\end{array}$ & 0.675 & $\begin{array}{c}0.348 \\
(0.097-1.248)\end{array}$ & 0.105 \\
\hline $\begin{array}{l}\text { Physical Activity } \\
<1 \text { time }\end{array}$ & $99(52.1 \%)$ & $72(37.9 \%)$ & $\begin{array}{c}1.566 \\
(0.912-2.690)\end{array}$ & 0.104 & $\begin{array}{c}1.041 \\
(0.358-3.027)\end{array}$ & 0.941 \\
\hline 1-2 times & $55(28.9 \%)$ & $77(40.5 \%)$ & $\begin{array}{c}.813 \\
(0.462-1.432)\end{array}$ & 0.475 & $\begin{array}{c}.685 \\
(0.236-1.988)\end{array}$ & 0.487 \\
\hline $\begin{array}{l}>3 \text { times } \\
\text { Smoking }\end{array}$ & $36(18.9 \%)$ & $41(21.6 \%)$ & $0 b$ & $0 \mathrm{~b}$ & $0 b$ & $0 \mathrm{~b}$ \\
\hline Ever & $88(46.3 \%)$ & $86(45.3 \%)$ & $\begin{array}{c}1.043 \\
(0.697-1.562)\end{array}$ & 0.837 & $\begin{array}{c}0.541 \\
(0.265-1.108)\end{array}$ & 0.093 \\
\hline Never & $102(53.7 \%)$ & $104(54.7 \%)$ & $0 \mathrm{~b}$ & $0 \mathrm{~b}$ & $0 \mathrm{~b}$ & $0 \mathrm{~b}$ \\
\hline
\end{tabular}

Odds ratio with $95 \%$ confidence interval, $\mathrm{BMI}=$ body mass index; The reference category is: $\mathrm{T} 1$. b: This parameter is reference category. P-value $<0.05$ significant

that was significantly associated with osteoporosis. Every unit change in the level of triglycerides was related toa0.004 unit decreased in osteoporosis. 
Table 6. Multiple Regression Analysis of the effect of risk factors of metabolic syndrome on T-score

\begin{tabular}{lcccc}
\hline Risk Factors & B & SE & $\beta$ & 0.014 \\
WC & 0.000 & 0.002 & -0.014 & 0.781 \\
SBP & 0.001 & 0.005 & 0.043 & 0.056 \\
DBP & 0.006 & 0.008 & -0.194 & 0.443 \\
TG & 0.004 & 0.001 & 0.019 & 0.001 \\
HDL & 0.002 & 0.005 & 0.023 & 0.729 \\
FBS & 0.001 & 0.002 & 0.664 \\
\hline
\end{tabular}

B; unstandardized beta, SE; standard error, $\beta$; standardized beta, WC; waist circumference, SBP; systolic blood pressure, DBP; diastolic blood pressure, TG; triglyceride, HDL-C; high density lipoprotein cholesterol, FBS; fasting blood sugar. P-value $<0.05$ significant.

\section{Discussion}

The study revealed that osteoporosis was significantly associated with metabolic syndrome in Saudi women. This association was unbiased of other covariates. This result is consistent with other studies (16-18). It is suggested that low levels of HDL are risk factors for osteoporosis (19-22).

This study found that metabolic syndrome components were more common in osteoporotic patients compared to non-osteoporotic patients. These results were similar to other studies which found that higher blood pressure in osteoporotic patients is associated with an increased chance of bone loss (23). Furthermore, low HDL levels were observed among those who had osteoporosis (24). A previous case-control study found that high blood pressure was a major contributor to bone loss. (25) Another component of MetS is the TG level. Our study found high levels of TG in osteoporotic patients and TG levels increased with age.

Results found that MetS is statistically related to $\mathrm{WC}$ and obesity. This result was consistent with other studies. Pathophysiological systems connecting fracture to obesity is not still determined. Growth of fat around the hip joint may lead to an elevated level of emission of proinflammatory cytokines that are harmful to bone. (26-28)

Physical activity was also associated with osteoporosis in this study. This result is in contrast with some other studies. A previous study found that those who were more physically active had a less chance of osteoporosis fracture (29).

The study also found that those women who covered their bodies had more osteoporosis. The reason for this association is that sunlight is an important factor for bone mineralization. This result is consistent with other studies (30-31).

The study had several limitations. This was a casecontrol study and information bias might be present in the result. Second, the subjects were selected from two centers only.

\section{Conclusion}

Our findings suggested a strong association between Mets and BMD. Early diagnosis of osteoporosis in those with MetS is important.

\section{Acknowledgment}

We should extend our sincere appreciation to the Department of Public Health, Imam Abdulrahman Bin Faisal University, for the provision of the necessary support that enabled us to fulfill this study.

\section{Research involving human participants}

All procedures performed in this study involving human participants were in accordance with the ethical standards of the institutional and/or national research committee and with the 1964 Helsinki declaration and its later amendments or comparable ethical standards. This research was approved by the ethical review committee of the hospital. Confidentiality of data has maintained.

\section{Conflict of Interests}

The authors declare that they have no competing interests.

\section{References}

1. Ranasinghe P, Mathangasinghe Y, Jayawardena R, Hills AP, Misra A. Prevalence and trends of metabolic syndrome among adults in the asia-pacific region: a systematic review. BMC Public Health, 2017;17(1): 101

2. The IDF consensus worldwide definition of the METABOLIC SYNDROME.2016. \{internet Available from: https://www.idf.org/component/attachments/attachments.html?id=705 \&task $=$ download

3. Wu Y, Ding Y, Tanaka Y, Zhang W. Risk factors contributing to type 2 diabetes and recent advances in the treatment and prevention. Int $\mathrm{J}$ Med Sci. 2014;11(11):1185-1200.

4. AlRubeaan K, Bawazeer N, Al Farsi Y, Youssef AM, Al-Yahya AA, AlQumaidi H,et al. Prevalence of metabolic syndrome in Saudi Arabia - a cross sectional study. BMC Endocr Disord. 2018;18(1):16.

5. Kim SH, Kim J. The Relationship between Risk Factors for Metabolic Syndrome and Bone Mineral Density in Menopausal Korean Women. Iran J Public Health. 2019;48(6):1025-1032.

6. Lin HH, Huang CY, Hwang LC. Association between metabolic syndrome and osteoporosis in Taiwanese middle-aged and elderly participants. Arch Osteoporos. 2018;13(1):3-5.

7. Wani K, Yakout SM, Ansari MGA, Sabico S, Hussain SD, Alokail $\mathrm{MS}$, et al. Metabolic Syndrome in Arab Adults with Low Bone Mineral Density. Nutrients. 2019;11(6): 1405.

8. Wong S, Chin KY, Suhaimi F, Ahmad F, Ima-Nirwana S. The Relationship between Metabolic Syndrome and Osteoporosis: A Review. Nutrients. 2016;8(6): 347.

9. Qin L, Yang Z, Zhang W, Gu H, Li X, Zhu L, et al. Metabolic syndrome and osteoporotic fracture: a population-based study in China. BMC Endocrine Disorders. 2016;16(1):4-5

10. Alissa EM, Alnahdi WA, Alama N, Ferns GA. Relationship between the Components of the Metabolic Syndrome and Measures of Bone Mineral Density in Post-Menopausal Women. J Diabetes Mellitus. 2014;4(2):155-164.

11. Alshaikh MK, Filippidis FT, Al-Omar HA, Rawaf S, Majeed A, Salmasi AM. The ticking time bomb in lifestyle-related diseases among women in the Gulf Cooperation Council countries; review of systematic reviews. BMC Public Health. 2017;17(1):536.

12. Loke SS, Chang HW, Li WC. Association between metabolic syndrome and bone mineral density in a Taiwanese elderly population. J Bone Min Metab. 2017; 36(2):200-208.

13. National plan for osteoporosis prevention and management in the kingdom of Saudi Arabia April 2018 \{internet\}\{cited 9/7/2019\}. 
Available from: https://www.moh.gov.sa/en/Ministry/MediaCenter/ Publications/Documents/NPOPM-2018.pdf

14. Osteoporosis and Musculoskeletal disorder, International Osteoporosis Fondation (IOF) \{internet\}. Avaliable from: https://www.iofbonehealth.org/osteoporosis-musculoskeletal-disorders

15. Wang D, Liu N, Gao Y, Li P, Tian M. Association Between Metabolic Syndrome and Osteoporotic Fracture in Middle-aged and Elderly Chinese Peoples. Cell Biochem Biophysics. 2014;70(2):12971303.

16. Iacobellis G, Iorio M, Napoli N, Cotesta D, Zinnamosca L, Marinelli $\mathrm{C}$, et al. Relation of adiponectin, visfatin and bone mineral density in patients with metabolic syndrome. J Endocrinol Invest. 2011;34:e1215 .

17. Manolagas SC, Almeida M. Gone with the Wnts: beta-catenin T-cell factor, forkhead box $\mathrm{O}$, and oxidative stress in age-dependent diseases of bone, lipid, and glucose metabolism. Mol Endocrinol. 2007;21:2605-14

18. McFarlane SI. Bone metabolism and the cardiometabolic syndrome:pathophysiologic insights. J Cardiometab Syndr. 2006;1:53-7.

19. Wimalawansa SJ. Vitamin D, in the new millennium. Curr Osteoporos Rep. 2012;10:4 15.

20. Navarro Mdel C, Saavedra P, Jódar E, Gómez de Tejada MJ, Mirallave A, Sosa M. Osteoporosis and metabolic syndrome according to socio-economic status; contribution of PTH, Vitamin D and body weight: The Canarian Osteoporosis Poverty Study (COPS). Clin Endocrinol (Oxf). 2012;78:681-6.

21. Parker J, Hashmi O, Dutton D, Mavrodaris A, Stranges S, Kandala $\mathrm{NB}$, et al. Levels of vitamin D and cardiometabolic disorders: systematic review and meta-analysis. Maturitas. 2010;65:225-36.

22. Hernández JL, Olmos JM, González-Macías J. Metabolic syndrome, fractures and gender. Maturitas. 2011;68:217-23.

23. Cappuccio FP, Meilahn E, Zmuda JM, Cauley JA. High blood pressure and bone-mineral loss in elderly white women: a prospective study. Study of Osteoporotic Fractures Research Group. Lancet. 2012;354:971-5.

24. Rejnmark L, Vestergaard P, Mosekilde L. Treatment with betablockers, ACE inhibitors, and calcium-channel blockers is associated with a reduced fracture risk: a nationwide case-control study. J Hypertens. 2016;24:581-9.

25. Vestergaard P, Rejnmark L, Mosekilde L. Hypertension is a risk factor for fractures. Calcif Tissue Int. 2019;84:103-11.

26. Hung J, McQuillan BM, Chapman CM, Thompson PL, Beilby JP. Elevated inteleukin-18 levels are associated with the metabolic syndrome independent of obesity and insulin resistance. Artheriscl Thromb Vasc Biol. 2015;25:1268-73.

27. Zuliani G, Volpato S, Galvani M, Blè A, Bandinelli S, Corsi AM, et al. Elevated C-reactive protein levels and metabolic syndrome in the elderly: The role of central obesity data from the InChianti study. Atherosclerosis. 2009;203:626-32.

28. Dai SM, Nishioka K, Yudoh K. Interleukin (IL) 18 stimulates osteoclast formation through synovial $\mathrm{T}$ cells in rheumatoid arthritis: comparison with IL1 beta and tumour necrosis factor alpha. Ann Rheum Dis. 2014;63:1379-86.

29. Leidig-Bruckner G, Ziegler R. Diabetes mellitus a risk for osteoporosis? Exp Clin Endocrinol Diabetes. 2011;109(2):493-514.

30. Leidig-Bruckner G, Grobholz S, Bruckner T, Scheidt-Nave C, Nawroth P, Schneider JG. Prevalence and determinants of osteoporosis in patients with type 1 and type 2diabetes mellitus. BMC Endocr Disord. 2014;14:33.

31. Zuliani G, Volpato S, Galvani M, Blè A, Bandinelli S, Corsi AM, et al. Dietary,calcium intake and risk of fracture and osteoporosis: prospective longitudinal cohort study. BMJ. 2011;342:d1473. 